\title{
A Description and Examination of Fluorescence in Nine North American Firefly Species (Coleoptera: Lampyridae)
}

\begin{abstract}
Aidan Wilcox
Tufts University, Boston, MA, USA

Correspondence should be addressed to Aidan Wilcox; aidanipw@gmail.com

Received 11 July 2020; Revised 24 November 2020; Accepted 15 January 2021; Published 28 January 2021

Academic Editor: Stephen W. Wilson

Copyright (c) 2021 Aidan Wilcox. This is an open access article distributed under the Creative Commons Attribution License, which permits unrestricted use, distribution, and reproduction in any medium, provided the original work is properly cited.

Fluorescence across the family Lampyridae has been documented sporadically but not comprehensively in formal research. Fireflies (Coleoptera: Lampyridae), best known for their bioluminescence, are also fluorescent. This fluorescence has been documented in several genera within the clade but is still an often overlooked aspect of firefly physiology in the common understanding of the species. To this end, the purpose of this study was to document and describe the fluorescence in nine species of North American fireflies, across three genera. Each species was photographed and a description of the fluorescent pattern was provided, as well as measurements of the specific spectral sensitivity of the fluorescent excitation and emission wavelengths. These data are intended to provide an identification guide of sorts to different firefly fluorescence, as well as documenting definitively its presence in several firefly genera.
\end{abstract}

\section{Introduction}

Fireflies (Coleoptera: Lampyridae) are charismatic insects most known for their bioluminescence, which is used to produce courtship signals by adults of many species $[1,2]$. It is believed that this trait originated phylogenetically in the larval stage $[3,4]$. Here, it is thought to have developed as an aposematic signal $[5,6]$ before being acquired as a trait in adults. Research has shown that, in addition to courtship, the bioluminescence of fireflies is detected by bats, who are thus deterred from preying upon fireflies [7]. Many firefly taxa are defended with chemicals known as lucibufagins [8-11], and their bioluminescence, along with other visual and auditory cues, serves as an aposematic signal in conjunction with their chemical defenses to facilitate predator learning and deter attacks.

Some fireflies are also known to display bright fluorescence [12-17]. Metcalf in 1943 first reported a red UV fluorescent pigment lampyrine in the firefly Photinus marginellus LeConte. Lampyrine was also noted in 43 different lampyrid taxa, while it was absent in the clades Phengodidae, Cantharidae, and Lycidae. Fluorescence has been described in other firefly species as well $[12,13,16,17]$, including Photinus, Ellychnia, and the newly described Oculogryphus chenghoiyanae [17].

Fluorescence is found in many biological systems whether as a by-product of other biological processes or a functional part of visual ecology. Fluorescence is found in many species, from humans (teeth) to stomatopods to pitcher plants [18]. Its presence in fireflies, while documented, still requires further study and precise measurement. Nine Nearctic firefly specimens from field sites in New England were collected and photographed under blue light to highlight their fluorescent patterning. Fluorescence was found in all species, with patterning varying from species to species. Here, the photographed specimens are presented, with descriptions of the fluorescent patterning. Excitation and emission spectra of fluorescence for each species were also measured and are presented. For many species shown here, this is the first instance of fluorescence documentation and description.

\section{Materials and Methods}

Fireflies were collected from various sites around the greater Boston area. Specimens were collected from Rock Meadow 
in Belmont (42.401, -71.197), Muster Field in Lincoln (42.422, -71.306), Smith Andover Field in Lincoln (42.436, -71.381), and Menotomy Rocks Park in Arlington (42.410, -71.169). Only male fireflies were collected. Each specimen was freeze-killed at $-20^{\circ} \mathrm{C}$ for 30 minutes and then stored in a dark, dry environment until ready to be examined. This was done to prevent either light or humidity from degrading the fluorescent pigment.

Specimens were then photographed with a Canon Rebel T2 Camera (Canon, Melville, NY). Specimens were photographed under white light, and under blue light using a yellow filter. Blue light was supplied by a NIGHTSEA Model SFA Royal Blue light emitting at 440-460 nm, using a NIGHTSEA yellow barrier filter (NIGHTSEA, Lexington MA) to screen reflected light and highlight fluorescent wavelengths.

A FluoroMax-2 spectrofluorometer (FluoroMax, Edison, NJ) was used to analyze the fluorescent excitation and emission spectra. Specimens were placed in a dark box devoid of light with the fluorescence probe on the pronotum, as close as possible to the specimen. The spectrofluorometer was used to measure the optimum emission spectra of the specimen, and this wavelength was then used to ascertain the specific excitation wavelength using the same machine. This was then repeated with the lantern. It was not possible to dissect individual tissues or fluorophores. Specimens were dissected to isolate male genitalia and confirm genus and species identity.

\section{Results}

3.1. Descriptions of Fluorescence. For fluorescent photographs on dorsal and ventral sides, refer to Figures 1 and 2.

3.1.1. Pyractomena linearis LeConte (Figures 1(a) and 1(b)). Weak fluorescence is observed on dorsal side, around elytral margins, and on pronotum. Elytral margins show thin green fluorescence, being thicker on the outer edges and not attaining pronotum edges. Pronotum itself has two weak fluorescent stripes on either side of a darker wedge shaped area, not attaining edges or apex of pronotum. Ventrally, it displays much stronger green fluorescence around ventral elytral margins and lantern. It is fluorescent along the margins of the fifth and sixth abdominal segments. The ventral side of the pronotum is fluorescent attaining apex, and eyes display green fluorescence. Some weak fluorescence appears on the legs.

3.1.2. Pyractomena dispersa Green (Figures 1(c) and 1(d)). Bright fluorescence appears across all of pronotum, silhouetting a dark arrow shaped patch in the center. Green fluorescence is also observed all along elytral margins, being wider distally and extending to the pronotum. Mesothorax is also strongly fluorescent. Some fluorescent glow appears in middle of the elytra, being stronger towards the apex. Dorsally, lantern is strongly fluorescent. Diffuse fluorescence can be seen across dorsal abdomen, primarily at the thoracic segment margins. Elytra appear strongly fluorescent along margins, diffusely proximally towards the apex. Ventral pronotum is light green fluorescent. Head is light green fluorescent, primarily dorsally around the eyes and mouth.

\subsubsection{Pyractomena angulata (Say) (Figures 1(e) and 1(f)).} Green fluorescence appears across pronotum, except for dark patches on margins and dark stripe in center proceeding from base and tapering to apex. Elytral margins show light fluorescence much wider distally, varying in width throughout outer margins. Elytral fluorescence attains partially the pronotum border and silhouettes the mesonotum. Ventrally, the elytral fluorescence is much wider anteriorly and along the anterior margins, before tapering towards the posterior margins. Lantern is bright fluorescent. Aedeagus is outlined in green fluorescence. Ventrally, the pronotum is weakly fluorescent at the apex. Some fluorescence is also observed along the eyes and head, and some weak fluorescence appears on the legs.

3.1.4. Photuris versicolor Fab. (Figures $1(g)$ and $1(h)$ ). On the dorsal side, the pronotum has two crescent shaped fluorescent stripes, silhouetting a pointed dark area wider basally and tapering towards but not attaining the apex. There is a diffuse fluorescence throughout the elytra, concentrated along the margins, but not sharply defined. Ventrally, the lantern displays prominent fluorescence, being easily the brightest area. The genitalia and the eighth abdominal segment are also fluorescent green. The underside of the pronotum is fluorescent, as well as the elytral margins. The legs are weakly fluorescent, especially at the joints.

3.1.5. Photinus obscurellus LeConte (Figures 2(a) and 2(b)). Diffuse fluorescence is observed throughout the pronotum, excepting an hourglass dark shape in the center. Fluorescence is concentrated along the pronotal margins. Bright fluorescence appears along elytral margins not attaining the pronotal border. On inner elytral margins, fluorescence extends to the apex, but tapers off anteriorly. Ventrally, lantern and aedeagus are bright fluorescent. Fluorescence extends fully around elytral margins and is diffuse throughout the pronotum and prothorax. Head and eyes show weak fluorescence. Note that specimen is speckled with bright fluorescent dots, primarily on the dorsal side. This may be a side effect of fluorescent particles such as pollen stuck in the exoskeleton.

\subsubsection{Photinus greeni Lloyd (Figures 2(b) and 2(c)).} Green fluorescence can be seen throughout the pronotum except for one dark pigmented stripe which runs centrally beginning at the basal end and approaching but not quite attaining the apex. Fluoresce appears along the elytral margins, being thicker on the distal edges and towards anterior ends and thin on the inner margins. The base of the elytra where they join the thorax is completely dark. Ventrally, the most prominent fluorescence is on the lantern organ, which is bright green. The genitalia were not visible or prominently fluorescent. The underside of the pronotum is 


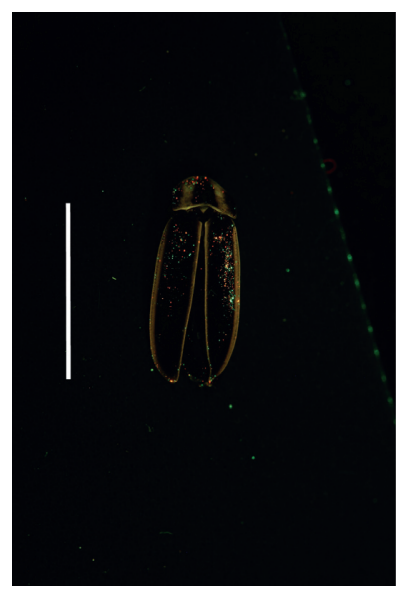

(a)

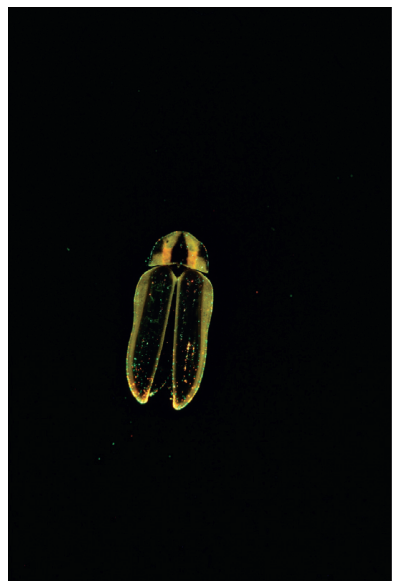

(e)

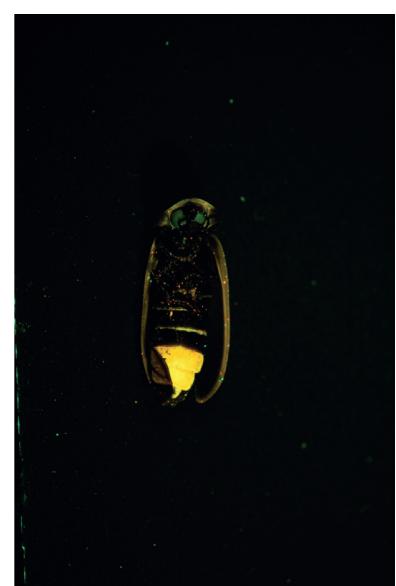

(b)

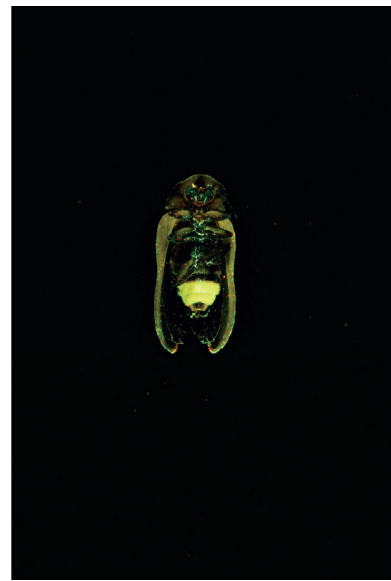

(f)

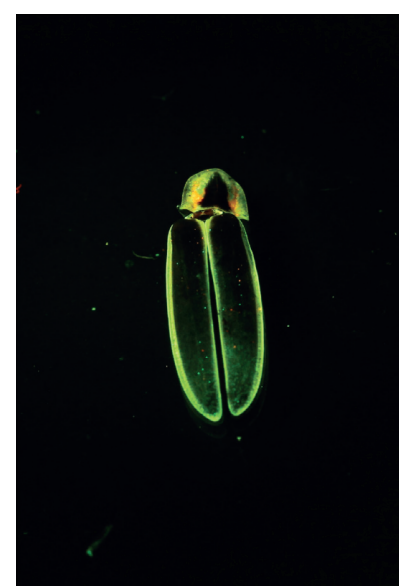

(c)

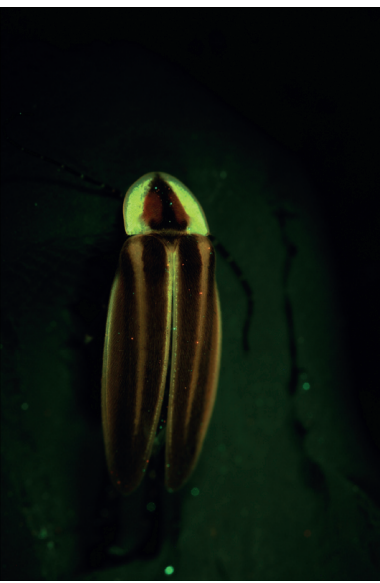

(g)

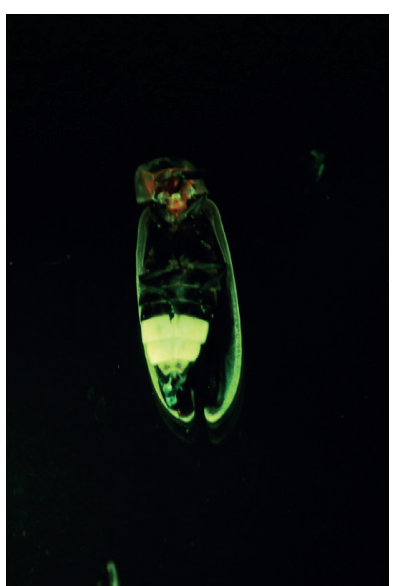

(d)

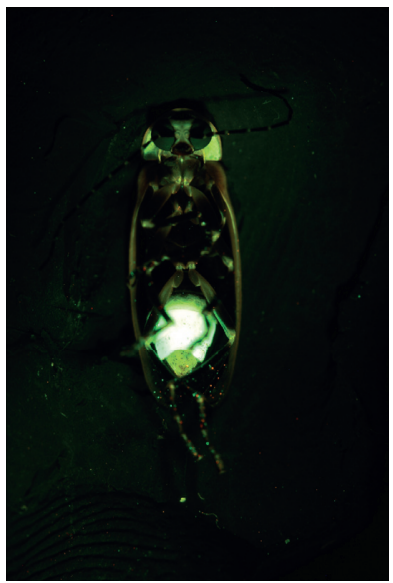

(h)

Figure 1: Dorsal and ventral fluorescence in Pyractomena linearis (a and b), Pyractomena dispersa (c and d), Pyractomena angulata (e and $\mathrm{f}$ ), and Photuris versicolor ( $\mathrm{g}$ and $\mathrm{h}$ ). Specimens were photographed under white light, and under blue light using a yellow filter. Blue light was supplied by a NIGHTSEA Model SFA Royal Blue light emitting at 440-460 nm, using a NIGHTSEA yellow barrier filter to screen. Scale bar represents approx. $15 \mathrm{~mm}$.

weakly green fluorescent, as are the outer elytral margins. A faint fluorescence is seen on the undersides of the legs, particularly the third pair of hind legs.

\subsubsection{Photinus ignitus Fall (Figures 2(e) and 2(f)). Bright} fluorescence is observed on pronotum, silhouetting a dark patch which begins narrowly before widening towards apex. Elytral margins are fluorescent, widening towards apex. Interior margins extend narrowly towards mesonotum. Lantern and aedeagus segments are brightly fluorescent, as well as pronotum. Fluorescence appears along the extent of the elytral margins, being diffuse towards the interior. Ventral pronotum and eyes are fluorescent, along with diffuse fluorescence around the leg joints.

3.1.8. Photinus aquilonius Lloyd (Figures 2(g) and 2(h)). Fluorescence can be seen across pronotum attaining the apex, with dark spot in the center, being wider towards but not reaching apex. Elytral fluorescence is concentrated on edges, extending diffusely a short distance into the interior of the elytra. Fluorescence along elytra extends fully to the pronotum but not across the attached edges of the elytra. Ventrally, abdominal segments from lantern posteriorly are fluorescent, with the lantern itself displaying bright fluorescence. Weaker fluorescence is also observed along elytral margins and pronotum margins, and weak fluorescence appears throughout legs.

3.1.9. Ellychnia corrusca L. (Not Pictured). E. corrusca adults showed fluorescence on the dorsal pronotum, which was limited to the curved areas of nonmelanized chitin bracketing the central dark spot. The area below and just posterior to the tip of the scutellum also fluoresced red. No fluorescence was apparent on the elytra. Ventrally, the pronotum and eyes showed green fluorescence, while soft tissues of the head and thorax displayed red fluorescence visible through the transparent integument. Patches of red fluorescence also surrounded the base of the legs. For further description of fluorescence in Ellychnia, refer to Wilcox and Lewis [19]. 


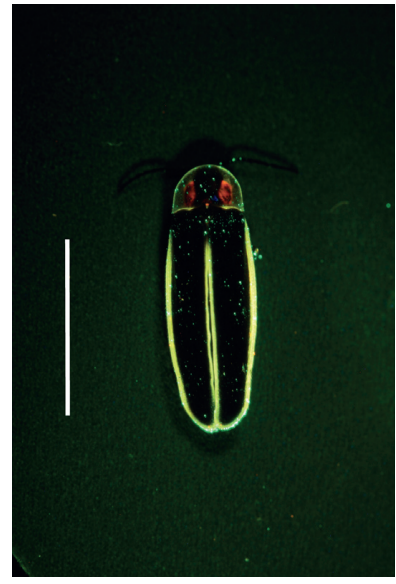

(a)

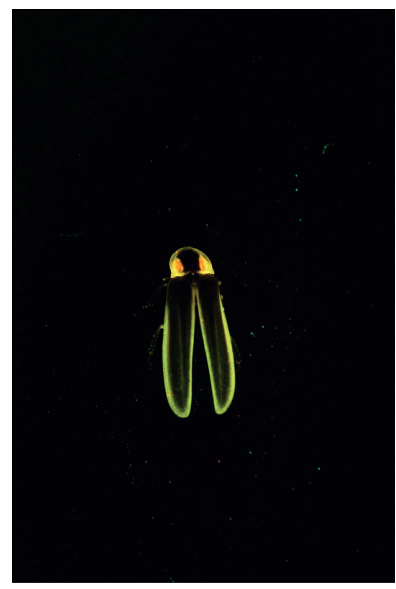

(e)

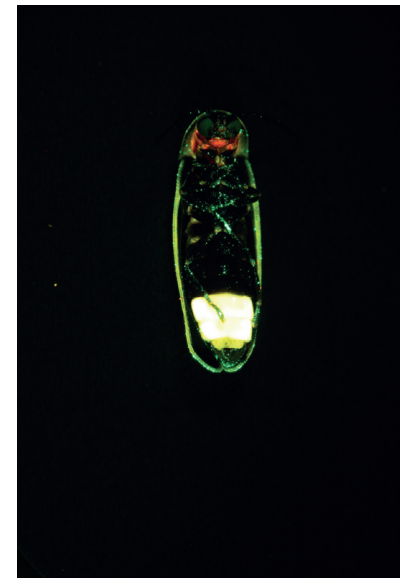

(b)

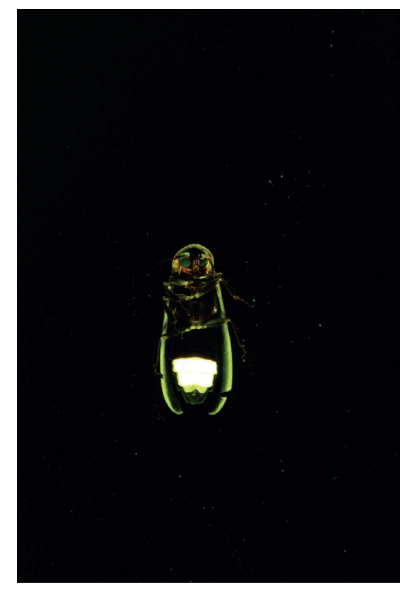

(f)

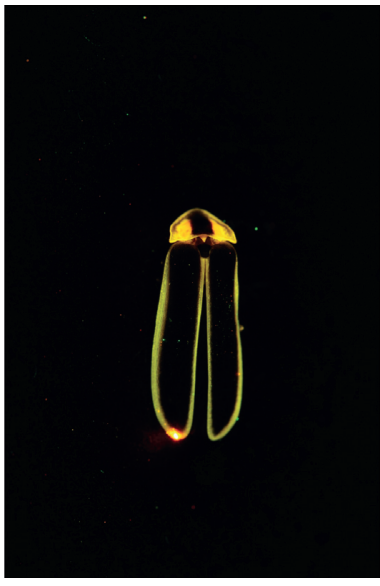

(c)

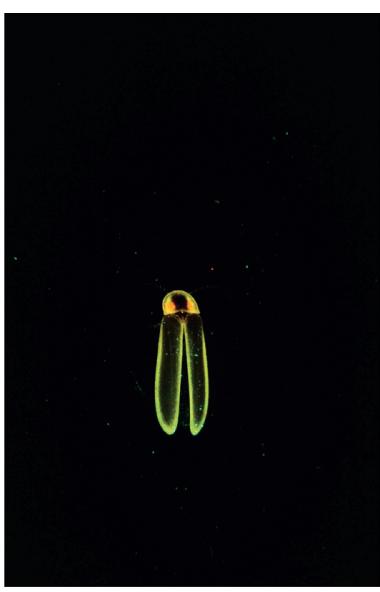

(g)

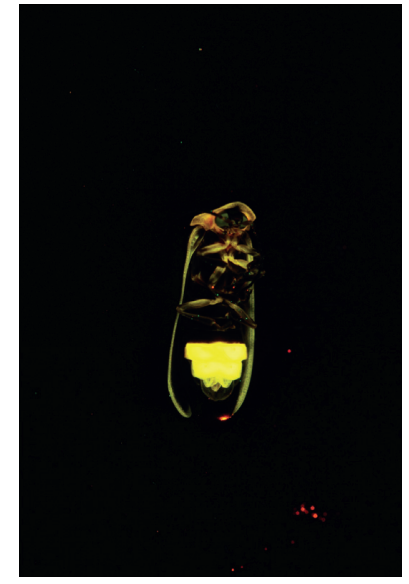

(d)

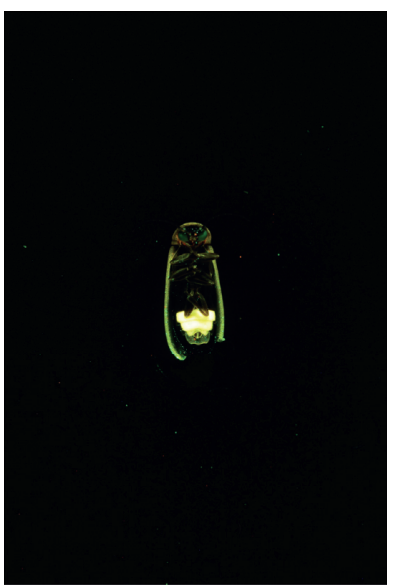

(h)

Figure 2: Dorsal and ventral aspects of Photinus obscurellus (a and b), Photinus greeni (c and d), Photinus ignites (e and f), and Photinus aquilonius ( $\mathrm{g}$ and $\mathrm{h}$ ). Specimens were photographed under white light, and under blue light using a yellow filter. Blue light was supplied by a NIGHTSEA Model SFA Royal Blue light emitting at $440-460 \mathrm{~nm}$, using a NIGHTSEA yellow barrier filter to screen. Scale bar represents approx. $15 \mathrm{~mm}$.

3.2. Wavelength Analysis. The majority of fireflies displayed fluorescence that was excited in the $350-450 \mathrm{~nm}$ range. Photinus greeni and Ellychnia corrusca had peak excitation values outside this range, at approximately $530 \mathrm{~nm}$. There was wide variation in the emission wavelengths of fluorescence detected on the pronotum, ranging from $400 \mathrm{~nm}$ in Photinus aquilonius to $600 \mathrm{~nm}$ in Photinus greeni.

Fluorescence in all but two species (E. corrusca and $P$. greeni) peaked in the UV to blue wavelengths, with a range of about $350-450 \mathrm{~nm}$. The remainder of species tested fluoresced under yellow/green light at approximately $530 \mathrm{~nm}$. There was no apparent pattern seen between genera.

Lantern fluorescence also varied. It was found that different species fluoresced across a range of 400-600 nm. There was no apparent pattern across genera, although generally Photinus species fluoresced at shorter wavelengths. The excitation peaks for firefly lanterns were fairly consistent, mostly between 350 and $450 \mathrm{~nm}$, with a few outliers. In some species ( $P$. linearis, $P$. angulata) multiple distinct excitation peaks at the same emission wavelength were noted. Refer to Table 1 for full details.
There was notable variation between two specimens collected, initially identified as being in the Pyractomena linearis species complex. These two specimens displayed remarkably different spectral patterns for both emission and excitation spectra.

\section{Discussion and Conclusions}

Without data on the contribution of fluorescence to the total visual signal under real world conditions, I cannot make definitive claims about the purpose or use of fluorescence in firefly sensory ecology [20]. However, the data collected here may help in identifying and classifying fireflies in the future. Previous studies, taken with the results described here, including spectral sensitivities, indicate that fluorescence as a trait is common in the family Lampyridae.

The difference in fluorescent wavelengths between the pronotum and the lantern of the same species would suggest that fluorescence is caused by multiple chemicals in fireflies or that light absorption and transmission are affected differently in different organs. In some species, the presence of 
TABLE 1: Excitation and emission peak values for all species tested.

\begin{tabular}{lcccc}
\hline Species & $\begin{array}{c}\text { Pronotum excitation } \\
\text { wavelength }\end{array}$ & $\begin{array}{c}\text { Pronotum emission } \\
\text { wavelength }\end{array}$ & $\begin{array}{c}\text { Lantern excitation } \\
\text { wavelength }\end{array}$ & $\begin{array}{c}\text { Lantern emission } \\
\text { wavelength }\end{array}$ \\
\hline Py linearis 1 & 372 & 500 & 535 & 590 \\
Py linearis 2 & 427 & 592 & 367 & 492 \\
Py angulata & 421 & 531 & $375-434$ & 535 \\
Py dispersa & 398 & 431 & 404 & 462 \\
Photuris & 368 & 466 & 364 & 505 \\
Ph obscurellus & 364 & 410 & 375 & 418 \\
Ph greeni & 537 & 601 & 370 & 424 \\
Ph ignitus & 436 & 587 & 468 & 539 \\
Ph aquilonius & 361 & 404 & 359 & 407 \\
E. corrusca & 536 & 581 & NA & NA \\
\hline
\end{tabular}

Values represent the wavelength in nanometers of the most intense peak for each test. Where a range or multiple values are reported, the peak was especially broad or contained multiple peaks. Ellychnia do not possess a lantern and so these values are left blank.

multiple excitation peaks would suggest that the chemical composition of the lantern involves multiple fluorescent chemicals, likely as a result of the chemiluminescent organs contained there, and may be different from that of the pronotum, in which fluorescence is seen in soft tissue gaps in opaque chitin. Another explanation could be frequency modulation of light through translucent exoskeletal tissue. Fluorescence seems to be prevalent throughout the soft tissue of the firefly. Wilkerson and Lloyd [21] reported fluorescence in ground up homogenates of firefly species, suggesting it may be prevalent throughout the soft tissue. Fluorescence has also been reported in the hemolymph of fireflies as they reflex-bleed [14], though no spectral data are available for this.

Fluorescence in fireflies has been documented previously as "red" or "green" [15, 17]. Visually, fireflies tend to present a pattern of both green and red in combination. These data seem to show that the green fluorescence in fireflies is more prevalent than the red. Unfortunately, the equipment did not allow for enough resolution to distinguish different parts of the fluorescent patterns, so it was not possible to differentiate those wavelengths. For this reason, it was also not possible to examine the elytral margins of specimens, as they presented a too small target. In any case, the green fluorescence seems to dominate the red one.

The variation found in the two $P$. linearis specimens is notable for individuals being ostensibly part of the same species. This may be an example of different species or subspecies within the linearis species complex, which encompasses several species or subspecies of Pyractomena to the point where differentiation by physiology or behavior is extremely difficult [22]. The same was found in Photinus aquilonius. However, as DNA testing was not possible, it was not feasible to definitively account for this variation. These data may point to a way to differentiate species of Pyractomena (and Photinus) without necessitating complete dissection.

While this study has successfully documented fluorescence in nine species of fireflies, it has not demonstrated that fluorescence represents a significant contribution to the overall visual signal. This is key, along with behavioral observations, in determining whether fluorescence is a selected-for trait or merely incidental to an organism's chemical processes $[18,20]$. Such an endeavor is beyond the scope of this study, but going forward this is an important avenue of research in firefly ecology. The fluorescence found here is certainly found in visually conspicuous areas and is excitable by wavelengths that fireflies are exposed to, supporting its role as a visual signal [18]. This alone however does not provide sufficient evidence of an evolutionary basis. Testing these two criteria will be important in determining the relevance of fluorescence to firefly behavior, and further study is suggested in this area. Fluorescence is a potentially important aspect of firefly physiology, and it is my hope that this and further studies will shed light on it (so to speak) as a future avenue of research.

\section{Data Availability}

The underlying data can all be found in the results of the manuscript.

\section{Conflicts of Interest}

The author declares that there are no conflicts of interest regarding the publication of this paper.

\section{Acknowledgments}

The author would like to thank Dr. Sara Lewis of Tufts University for her advice and support in this project, Dr. Charles Mazel for photography and technical assistance, and Ms. Alex Muccio for assistance in copy editing this manuscript.

\section{References}

[1] J. Lloyd, "Family 62: lampyridae," in American Beetles, vol. 2, pp. 187-196, CRC Press, Boca Raton, FL, USA, 2002.

[2] J. Lloyd, "Fireflies (Coleoptera: Lampyridae)," in Encyclopedia of Entomology, J. Capinera, Ed., pp. 1429-1452, Springer, Berlin, Germany, 2008.

[3] M. A. Branham and J. W. Wenzel, "The origin of photic behavior and the evolution of sexual communication in fireflies (Coleoptera: Lampyridae)," Cladistics, vol. 19, no. 1, pp. 1-22, 2003.

[4] G. J. Martin, M. A. Branham, M. F. Whiting, and S. M. Bybee, "Total evidence phylogeny and the evolution of adult 
bioluminescence in fireflies (Coleoptera: Lampyridae)," Molecular Phylogenetics and Evolution, vol. 107, pp. 564-575, 2017.

[5] R. De Cock and E. Matthysen, "Glow-worm larvae bioluminescence (Coleoptera: Lampyridae) operates as an aposematic signal upon toads (Bufo bufo)," Behavioral Ecology, vol. 14, no. 1, pp. 103-108, 2003.

[6] T. J. Underwood, D. W. Tallamy, and J. D. Pesek, "Bioluminescence in firefly larvae: a test of the aposematic display hypothesis (Coleoptera: Lampyridae)," Journal of Insect Behavior, vol. 10, no. 3, pp. 365-370, 1997.

[7] B. C. Leavell, J. J. Rubin, C. J. W. McClure, K. A. Miner, M. A. Branham, and J. R. Barber, "Fireflies thwart bat attack with multisensory warnings," Science Advances, vol. 4, no. 8, pp. 1-6, 2018.

[8] T. Eisner, M. A. Goetz, D. E. Hill, S. R. Smedley, and J. Meinwald, "Firefly "femmes fatales" acquire defensive steroids (lucibufagins) from their firefly prey," in Proceedings of the National Academy of Sciences, vol. 94, no. 18, pp. 9927-9728, 1997.

[9] M. A. Goetz, J. Meinwald, and T. Eisner, "Lucibufagins, IV. new defensive steroids and a pterin from the firefly, Photinus pyralis (Coleoptera: Lampyridae)," Experientia, vol. 37, no. 7, pp. 679-680, 1981.

[10] S. R. Smedley, R. G. Risteen, K. K. Tonyai et al., "Bufadienolides (lucibufagins) from an ecologically aberrant firefly (Ellychnia corrusca)," Chemoecology, vol. 27, no. 4, pp. 141-153, 2017.

[11] J. Tyler, W. McKinnon, G. A. Lord, and P. J. Hilton, "A defensive steroidal pyrone in the glow-worm Lampyris noctiluca L. (Coleoptera: Lampyridae)," Physiological Entomology, vol. 33, no. 2, pp. 167-170, 2008.

[12] J. M. Cicero, "Ontophylogenetic character analysis of Diaphanes (Coleoptera: Lampyridae) and extrapolation to the broader coleoptera," The Pan-Pacific Entomologist, vol. 84, no. 3, pp. 200-219, 2008.

[13] D. D. Deheyn and L. A. Ballantyne, "Optical characterization and redescription of the South Pacific firefly bourgeoisia hypocrita olivier (Coleoptera: Lampyridae: Luciolinae)," Zootaxa, vol. 2129, no. 1, pp. 47-62, 2009.

[14] F. A. McDermott, "Luciferesceine, ${ }^{2}$ the fluorescent material present in certain luminous insects," Journal of the American Chemical Society, vol. 33, no. 3, pp. 410-416, 1911.

[15] R. L. Metcalf, "The isolation of a red-fluorescent pigment, lampyrine, from the Lampyridae1," Annals of the Entomological Society of America, vol. 36, no. 1, pp. 37-40, 1943.

[16] A. Sannasi, "Resilin in the lens cuticle of the firefly, photinus pyralis linnaeus," Experientia, vol. 26, no. 2, p. 154, 1970.

[17] V. Yiu and M.-L. Jeng, "Oculogryphus chenghoiyanae sp. N. (coleoptera, lampyridae): a new ototretine firefly from Hong Kong with descriptions of its bioluminescent behavior and ultraviolet-induced fluorescence in females," ZooKeys, vol. 739, no. 739, pp. 65-78, 2018.

[18] M. G. Lagorio, G. B. Cordon, and A. Iriel, "Reviewing the relevance of fluorescence in biological systems," Photochemical and Photobiological Sciences, vol. 14, no. 9, pp. 1538-1559, 2015.

[19] A. Wilcox and S. Lewis, "Fluorescence in fireflies (Coleoptera: lampyridae): using sentinel prey to investigate a possible aposematic signal," Florida Entomologist, vol. 102, no. 3, p. 614, 2019.

[20] C. Mazel, "Method for determining the contribution of fluorescence to an optical signature, with implications for postulating a visual function," Frontiers in Marine Science, vol. 4, 2017.

[21] R. C. Wilkerson and J. E. Lloyd, "The application of paper chromatography of fluorescent cmpounds to the systematics of fireflies (Coleoptera, Lampyridae)," The Coleopterists Bulletin, vol. 29, no. 4, pp. 339-348, 1975.

[22] J. W. Green, "Revision of the nearctic species of Pyractomena (Coleoptera: Lampyridae)," The Wasmann Journal of Biology, vol. 15, no. 48, pp. 237-284, 1957. 